EPJ Web of Conferences 78, 03001 (2014)

DOI: $10.1051 /$ epjconf / 20147803001

(C) Owned by the authors, published by EDP Sciences, 2014

\title{
Describing pair production in inhomogeneous external fields
}

\section{with the Dirac-Heisenberg-Wigner formalism}

\author{
Dániel Berényi ${ }^{1,2, a}$, Sándor Varró ${ }^{3}$, Péter Lévai $^{2}$, and Vladimir V. Skokov ${ }^{4}$ \\ ${ }^{1}$ Loránd Eötvös University, $\mathrm{H}-1117$, Budapest, Hungary \\ ${ }^{2}$ Wigner RCP, Institute for Particle and Nuclear Physics, P.O. Box 49, Budapest 1525, Hungary \\ ${ }^{3}$ Wigner RCP, Institute for Solid State Physics and Optics, P.O. Box 49, Budapest 1525, Hungary \\ ${ }^{4}$ Department of Physics, Western Michigan University, 1903 W. Michigan Avenue, Kalamazoo, MI 49008
}

\begin{abstract}
In this contribution we present results on pair production from vacuum in QED in case of inhomogeneous external electric fields. The central tool of our description is the relativistic one particle single-time Wigner-function, that describes the evolution of the $e^{+} e^{-}$densities in phase-space. We compare the influence of homogeneous and inhomogeneous external fields, and find that the inhomogeneity near the Compton-scale increases particle production proportionally to the duration of the electric pulse. We also find, that when the inhomogeneity is restricted to a single direction, a significant component of the pair yield originates from the edge or surface where the electric field gradients are large, in agreement with the prediction of Heisenberg.
\end{abstract}

\section{Introduction}

In the recent years pair production from vacuum gained renewed interest from both theoreticians and experimentalists. If it is really observed then it would validate our understanding of nature on the nonlinear QED scale. With the advent of high intensity laser technologies experimentalists hope that the necessary field intensities will be reached in the near future. Meanwhile in the tunnels of the particle accelerators extremely energetic processes are created in the form of nucleon-nucleon collisions, and a successful model for the early stage of these came also in the form of pair production, but this time in the framework of QCD. To improve our understanding of these processes more and more detailed theoretical investigations needed.

In this work we focus on the phase-space description of pair production and show some remarkable differences between the space homogeneous and space inhomogeneous pair yields.

\section{Theoretical Framework}

Since pair creation is an inherently quantum phenomena we chose the equal-time single-particle Wigner function to represent the $e^{+} e^{-}$densities in phase-space. This description has the advantage that -after some approximations such as gradient expansion- it results in a differential equations system for the time evolution of the Wigner function and can include arbitrary external field dependence. The

\footnotetext{
ae-mail: berenyi.daniel@wigner.mta.hu
} 
formalism for QED is introduced in [1,2]. In the general case it is a 16 component partial differential equation system, but when only space homogeneous electric fields are present the evolution equations simplifies to a 3 component ordinary differential equation system, the Quantum Kinetic equations. From the 16 component Wigner-function the phase-space energy density $\epsilon$ can be calculated, which is related to the $f$ pair density in the Kinetic equations by $f=\frac{\epsilon}{4 \omega}+\frac{1}{2}$. We use $f$ to quantify the production yields.

We solve the evolution equations from pure vacuum initial conditions and chose the following electric field linearly polarized in th $z$ direction to produce pairs:

$$
E(x, t)=\frac{e_{z} E_{0}}{\cosh (t / \tau)^{2}} \frac{1}{2}\left(1-\tanh \left(\frac{x+R}{r}\right) \tanh \left(\frac{x-R}{r}\right)\right)
$$

The time dependent part is the so called Sauter-field for which an analytical solution is known when there is no space inhomogeneity [3]. We modulate this known time dependence with a plateau like space dependence in the $x$ direction. On one hand it serves as a reference because at the center of the plateau the field is almost homogeneous so we expect convergence to the known analytic results. On the other hand by changing the width $(R)$ of the plateau we are scaling the interaction volume and study space-localization induced by the gradients at the edges.

There is still another motivation for this kind of field: the non-Abelian pair production found to be strongly Abelian-dominated [4]. This means that the integrated particle yields from SU(2) for example are only different from the U(1) QED yields by a constant factor. Henceforth the characteristics of QED pair creation may be applied in the realm of QCD also. In heavy ion collisions pairs come from color strings and with this particular field configuration one may model a $1 \mathrm{D}$ cross-section of such a string.

\section{Results}

First we give a phase-space view of the asymptotic pair density $f$ on Figure 1. left. It is clear that while there is a bulk pair production in the spatial range $0-4 \lambda_{c}$, a higher amplitude more localized source also manifests at the edge of the plateau field.

Next, we turn to the integrated pair density in the transverse coordinate and longitudinal momentum taken at zero transverse momenta:

$$
n_{L}=\int_{-\infty}^{\infty} \mathrm{d} x \frac{\mathrm{d} p_{z}}{2 \pi} f\left(x, p_{x}=0, p_{y}=0, p_{z}\right) .
$$

We show our results comparing the homogeneous yield with the same spatial modulation to the truly inhomogeneous calculation. The top right plot of Figure 1. shows the time dependence while the other field parameters are kept fixed: $E_{0}=0.5 E_{c r}, R=5 \lambda_{c}, r=\lambda_{c}$, where $E_{c r}=\frac{m^{2} c^{3}}{e \hbar} \approx 1.32 \cdot 10^{18} \frac{\mathrm{V}}{\mathrm{m}}$ is the Schwinger-limit of electron-positron pair production in constant and uniform electric field. We find that for very short times there is no difference between the two cases: the inhomogeneity has no time to produce additional pairs, but if the pulse lasts longer, the inhomogeneous yield starts to significantly overcome the homogeneous one.

On the bottom right plot of Figure 1. the plateau width $(R)$ is changed for both cases and we find that the slopes of the curves are almost the same. While the linear scaling of the interaction volume with $R$ is expected, the constant difference between the two models is the genuine effect of the inhomogeneity. Taking into consideration the phase-space distribution of Figure 1. We draw the 

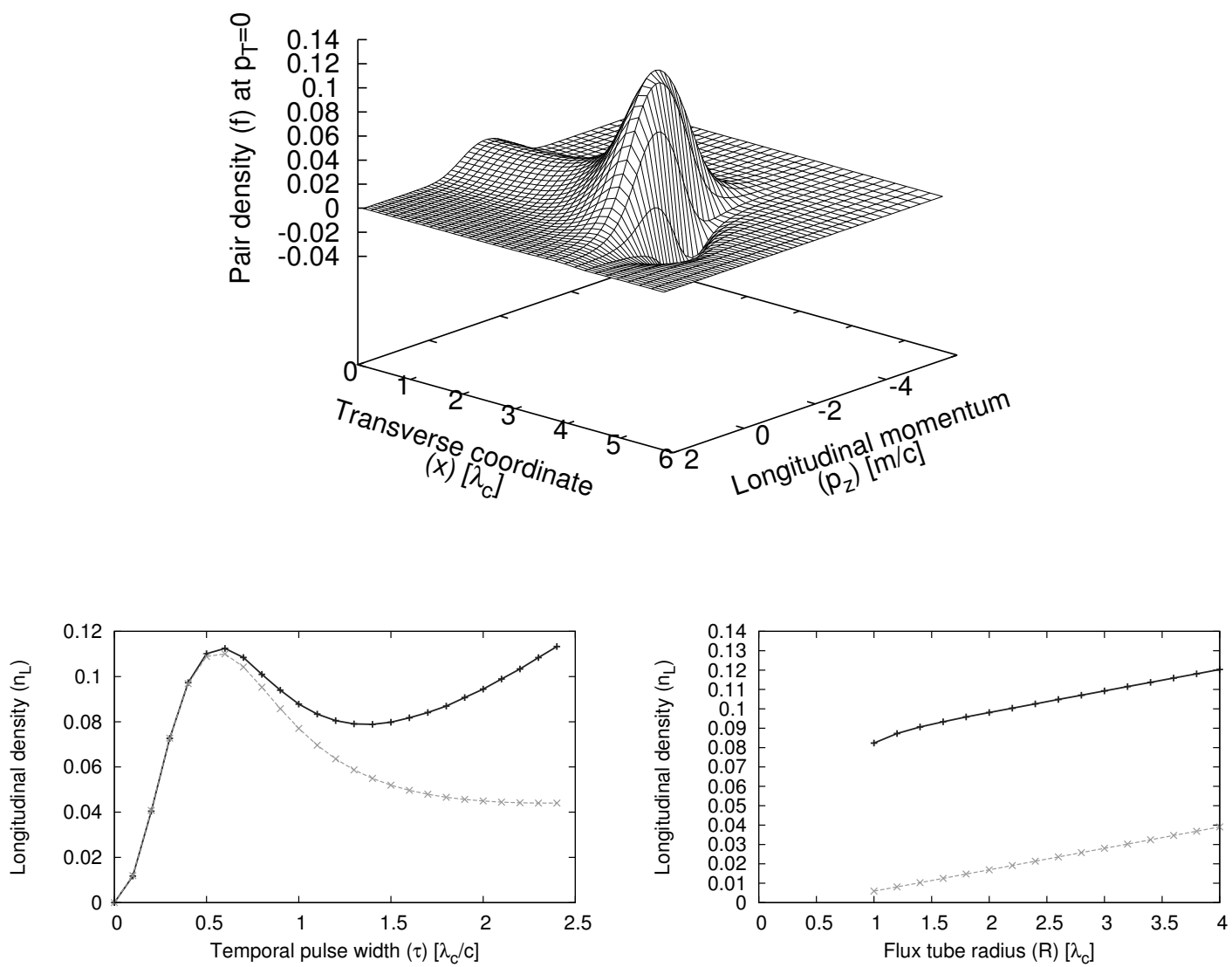

Figure 1. Left: phase-space view of the asymptotic pair density with parameters: $E 0=0.75 E_{c r}, R=4 \lambda_{c}, r=$ $0.5 \lambda_{c}, \tau=2 \lambda_{c} / c$. Note that the E field has the steepest gradient at $x=4 \lambda_{c}$. Right: Comparison of the particle yield in the inhomogeneous model (solid black line) with the homogeneous reference (dashed grey) for top: pulse width $(\tau)$ dependence and bottom: flux tube radius $(R)$ dependence.

conclusion that large number of the pairs are produced at the edge of the plateau or -in the string picture- at the edge of the color flux tube. Interestingly this in accordance with the observation that was made by Heisenberg during the study of the probability density of a fluctuating charge, namely that it is proportional to the surface of the interaction volume: $\propto V^{\frac{2}{3}}$ [5].

\section{Conclusion}

We compared the homogeneous and inhomogeneous pair production yields in an external electric field that is an Abelian analogue of the QCD color string. We found that close to the Compton scales significant part of pair production is happening near the edge of the string and may have an important contribution to the total number of particles produced and the observable spectra. Further details will be discussed in a forthcoming paper. 
EPJ Web of Conferences

This work was supported by the OTKA Grants No. 77816, No. 104260, No. 106119.

\section{References}

[1] I. Bialynicki-Birula, P. Gornicki, J. Rafelski, Phys. Rev. D44, 1825-1835. (1991)

[2] F. Hebenstreit, R. Alkofer, H. Gies, Phys. Rev. D82 105026. (2010)

[3] S. I. Kruglov, Radiat. Phys. Chem. 75, 723-728. (2006)

[4] V. V. Skokov and P. Levai, Phys. Rev. D78, 054004. (2008)

[5] W. Heisenberg, Sachsische Akademie der Wissenschaften, 86, 317. (1934) 